\title{
Foam Sclerotherapy and Laser Ablation of Varicose Veins: Newer Option in Bangladesh Perspective
}

\author{
Motiur Rahman Sarkar ${ }^{1}$, Nazmul Hosain ${ }^{2}$, Fazle Maruf ${ }^{2}$, M A Quaiyum Chowdhury², \\ Anisuzzaman², G M Mokbul Hossain ${ }^{3}$ \\ ${ }^{1}$ Department of Vascular Surgery, Chittagong Medical College \& Hospital, Chittagong, \\ ${ }^{2}$ Department of Cardiac Surgery, Chittagong Medical College \& Hospital. Chittagong, \\ ${ }^{3}$ Department of Vascular Surgery, NICVD, Dhaka
}

\begin{abstract}
Key wards: Varicose vein, Laser ablation, Saphenous vein.

Abstract

Background: Varicose vein is one of the most common vascular conditions in the adult population. These are conventionally treated with surgical high ligation of Sapheno-femoral junction and stripping of Great Saphenous Vein (GSV) with multiple phlebectomy. The aim of this study was to highlight the technical considerations in performing endovenous laser ablation of varicose vein, understanding the importance and role of Duplex Machine and Tumescent anesthesia with evaluation of outcome.

Methods: In this retrospective study we examined the records of 297 limbs of 220 patients operated at Vascular Care Centre at Dhaka, Bangladesh between October 2013 and October 2015. Among them 1 year follow up was performed in 118 patients. Patients were contacted through telephone calls and mobile SMSs with requests for follow up.

Results: Vast majority of the patients had a smooth recovery. Significant pain was present in only $10 \%$ of patients for 1week after laser therapy and $3 \%$ of patients noted bruising in thigh for 2 weeks. Among the 118 patients who reported for follow up at 12 months, $113(96 \%)$ showed effective persistent occlusion of GSV.

Conclusion: EVLA of varicose vein is gaining popularity day by day in Bangladesh. It has become established as a safe and effective treatment option for varicosities caused by GSV incompetence. Foam sclerotherapy with Sodium tetradecyl sulphate replacing the multiple phlebectomy may be a suitable adjunct. Quick return to normal activities, fewer wound infections, less leg pain and leg bruising are strongly associated with these endovenous techniques compared with Saphenous vein stripping.
\end{abstract}

(Cardiovasc. j. 2018; 10(2): 140-144)

\section{Introduction:}

Varicose vein is one of the most common vascular conditions in the adult population. Symptoms ranged from dilated and tortuous veins with fatigue, heaviness, and itching to more serious conditions such as skin discolouration and leg ulceration. ${ }^{1}$ Multiple techniques for treating saphenous reflux have been developed over the years, such as high ligation at saphenofemoral junction, stripping of great saphenous vein, ultrasonogram guided sclerotheraphy, as well as various combinations of these procedures. Recently, endovenous laser ablation identified as a viable treatment option for patients with saphenous reflux. ${ }^{2}$ Surgical treatment includes high ligation of the saphenous vein or high ligation and stripping of GSV. High ligation alone demonstrated a $43 \%$ - $71 \%$ failure rate at 5years and high ligation and stripping of GSV had a 25\%-60\% failure rate, ranging from 5-34 years in the available literature. ${ }^{3-6}$ The failures associated with high ligation alone stemmed from neovascularization that occurred in the saphenofemoral surgical bed and was attributed to the process of skeletenization and ligation of all tributaries of the saphenoferal junction. In the recurrences that occurred after stripping, the etiology may be incomplete removal of the entire vein. ${ }^{2}$ The most common complication of high ligation and GSV stripping is paresthesia with others like infection, haematoma, bruising. Because of high failure rates and recurrences of

Address of correspondence: Dr. Md. Motiur Rahman Sarkar, Assistant Professor, Vascular Surgery, Chittagong Medical College \& Hospital, Chittagong, Bangladesh. Email: drmotiurvs@gmail.com 
high ligation and stripping, there has long been interest in identifying a more effective and less invasive treatment option. , $^{7}$ The procedure called sclerotherapy or injection of varicose veins is designed to improve the appearance of your varicose veins. The veins are injected with a solution called a sclerosant which damages the internal lining of the vein and causes blood clotting within the vein. Over a period of time our own body will destroy the vein and it will disappear. The solution used is available in different concentrations depending on the size of the vein being treated and is called Sodium Tretradecyl Sulphate or "STD" for short. Foam sclerotherapy involves rapidly mixing volumes of the solution with a small volume of air producing a foam. The initial results with foam sclerotherapy are very promising and this method of treatment offers a possible alternative to surgery without the anaesthetic risk. However, it should be emphasized that this is a new treatment and at present the long term results are not yet known and it is not possible to say how this treatment compares in terms of results with conventional surgery or sclerotherapy.

Endovenous Laser ablation (EVLA) was first described by Bonne 1999 and has been available in Bangladesh since 2013. The laser differs primarily in their wavelengths. In our setup we are using $980 \mathrm{~nm}$ wave length. Although the initial success rate and short term durability of laser treatment for saphenous reflux are very promising, the published literature suffers from small number of patients and limited long-term follow-up for the different wavelength devices. Laser data are exclusively single centre data. This single centre studies demonstrate a very high initial success rate in the $95-100 \%$ range, with patients demonstrating approximately $95 \%$ persistence of occlusion of GSV after 12 months. Significant pain was present in $30 \%$ of patients for approximately 1 week after laser therapy, and up to $7 \%$ of patients were noted bruising in thigh for 2 weeks. ${ }^{9}$

Objectives of our study were-1) to understand the technical considerations in performing endovenous laser ablation of varicose vein, 2) To understand the importance and role of
Duplex Machine and Tumescent anesthesia 3) Outcome of laser ablation.

\section{Methods:}

In this retrospective study we examined the records of 297 limbs of 220 patients operated at Vascular Care Centre at Dhaka, Bangladesh between October 2013 and October 2015. Among them 1 year follow up was performed in 118 patients. Vascular Care Center has a good record keeping and organized patient follow up system. Patients were contacted through telephone calls and mobile SMSs with requests for follow up.

\section{Technique}

Complete informed written consent obtained before beginning the procedure. The common risk associated with laser includes transient paresthesia and bruising along the ablation track. Though there is no case of DVT and skin burn but chance of occurrence less than 1\% literature said. Vein mapping was done from the saphenofemoral junction through below knee. In our set up spinal anesthesia was given but worldwide tumescent anesthesia was used. Duplex guided venipuncture and vascular sheath was introduced in antegrade direction. Standard guidewires were used. For greatest accuracy, the saphenofemoral junction, the epigastric vein, and the laser tip was identified with longitudinal duplex imaging. Tumescent anesthesia (dilute mixture of lidocaine and normal saline) was then administered along the entire length of GSV within the perivenous space so that GSV deepened and collapsed. After administration of tumescent anesthesia, the entire course of GSV was evaluated with duplex to confirm that it was completely collapsed and away from the skin at least $1 \mathrm{~cm}$ deep and also surrounded by fluid in all levels. Laser tip was kept $2 \mathrm{~cm}$ distal from the saphenofemoral junction. The catheter fiber was then energized and withdrawn through the vein. The rate of pull back was adjusted to maintain an energy transfer of 80-100 jules/cm within the vein. We had used continuous energy production of 6.5 to 7.5 watts but most centres use 12-14 watts for energy production. After the fibre had been withdrawn to the venotomy site, the saphenous vein was again evaluated with duplex to identify vein wall thickening, concentric narrowing and absence of flow. 

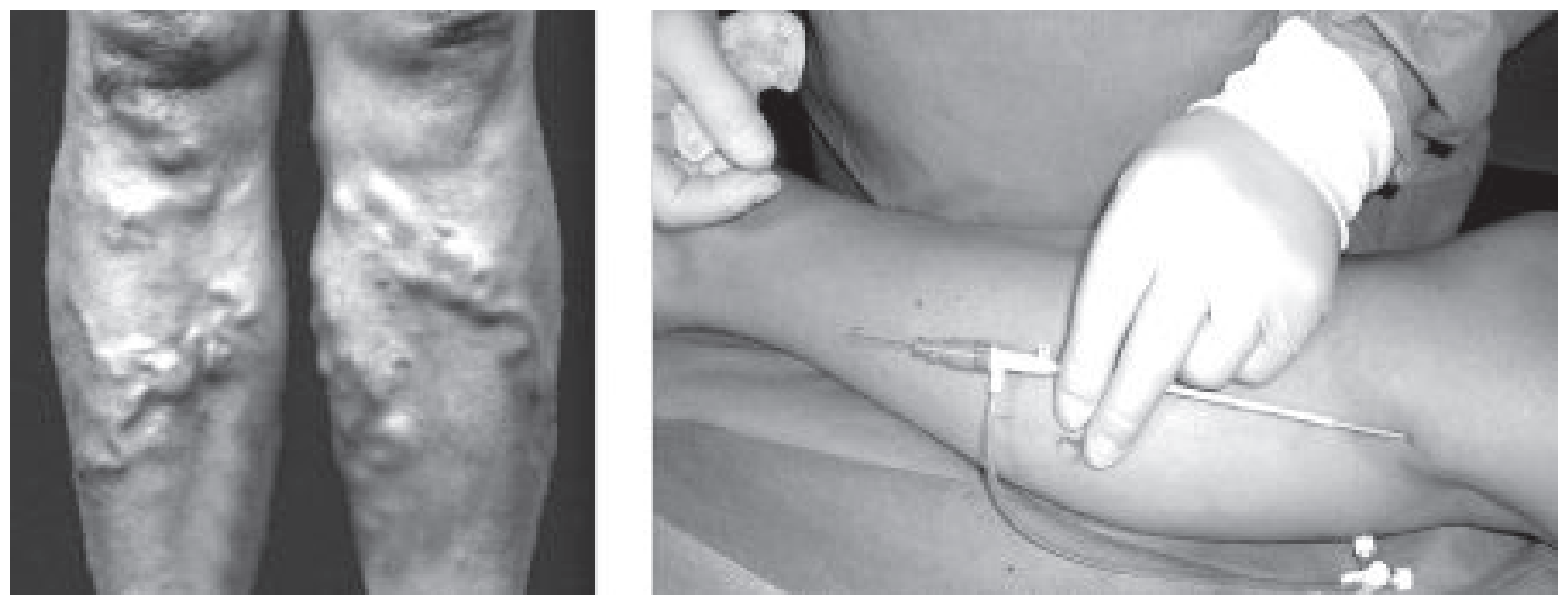

Fig.-1: A. Bilateral varicose veins \& 1B. Introduction of sheath.

The common femoral vein was also evaluated for compressibility and absence of thrombus. Gentle compression used in every case with crepe bandage up to $2^{\text {nd }}$ POD and then compression stocking for one week that matched with other centers. A follow up duplex examination was performed on 2-3 days after the laser to confirm a successful obliteration procedure and to rule out any potential DVT or extension of thrombus from the saphenous vein into the femoral vein.

\section{Foam Sclerotherapy}

Using ultrasound scanning, the main surface vein to be treated was marked on patient leg. The surgeon then injected to a small area of skin, usually the mid calf or lower thigh and a needle was placed into the vein. The needle was then flushed with a blood thinning agent containing heparin. Then 2 or 3 smaller needles were inserted into the visible varicose vein in the leg and the flushing procedure repeated. Leg was raised and the foam solution was injected in small volumes at a time into each of the needles. During this procedure patient was asked to bend his ankle up and down in order to increase the blood flow in deep veins. As the foam was injected patient may experience some slight stinging, but it was usually painless. The passage of the foam in the vein was monitored by the ultrasound scan and the foam injection into each needle was repeated 2 or 3 times. Once enough foam had been injected, the needles was removed, pieces of sponge was applied followed by a bandage in order to compress the treated veins. An elastic compression stocking was then to be put on patient's leg, including the thigh with a waist attachment. This will feel tight but should not be so tight as to make the foot painful or discolored. Following treatment the sponge, bandage and stocking were kept for 5 days.
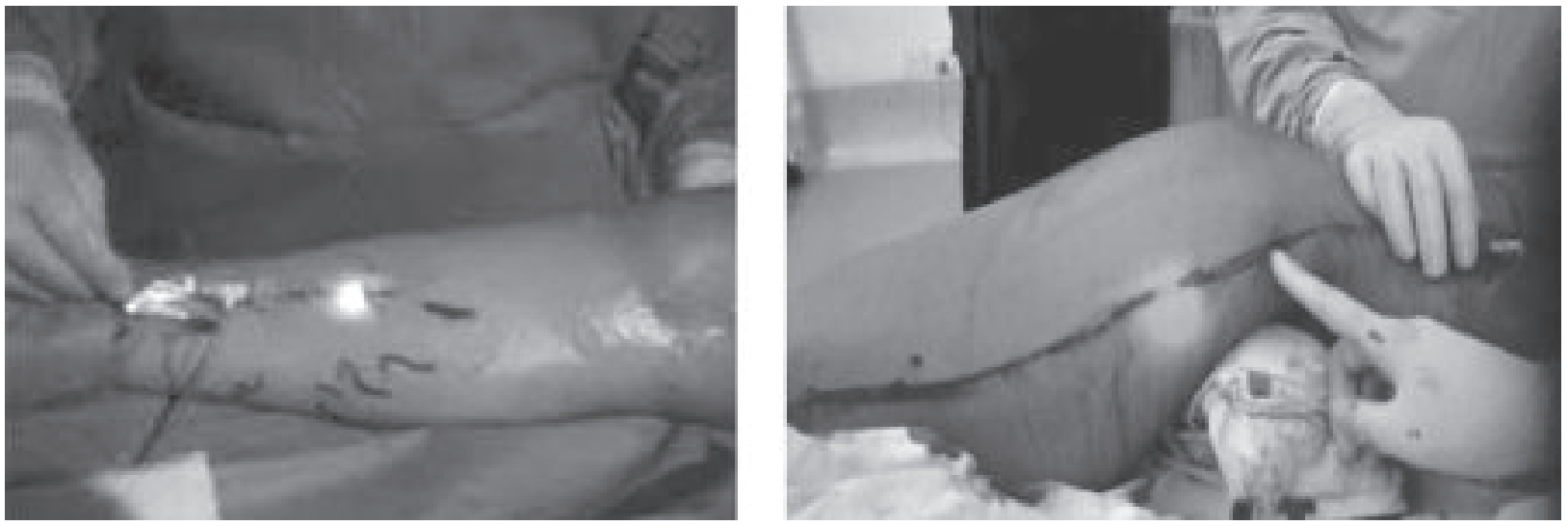

Fig 2A \& B: Laser ablation in progress. 

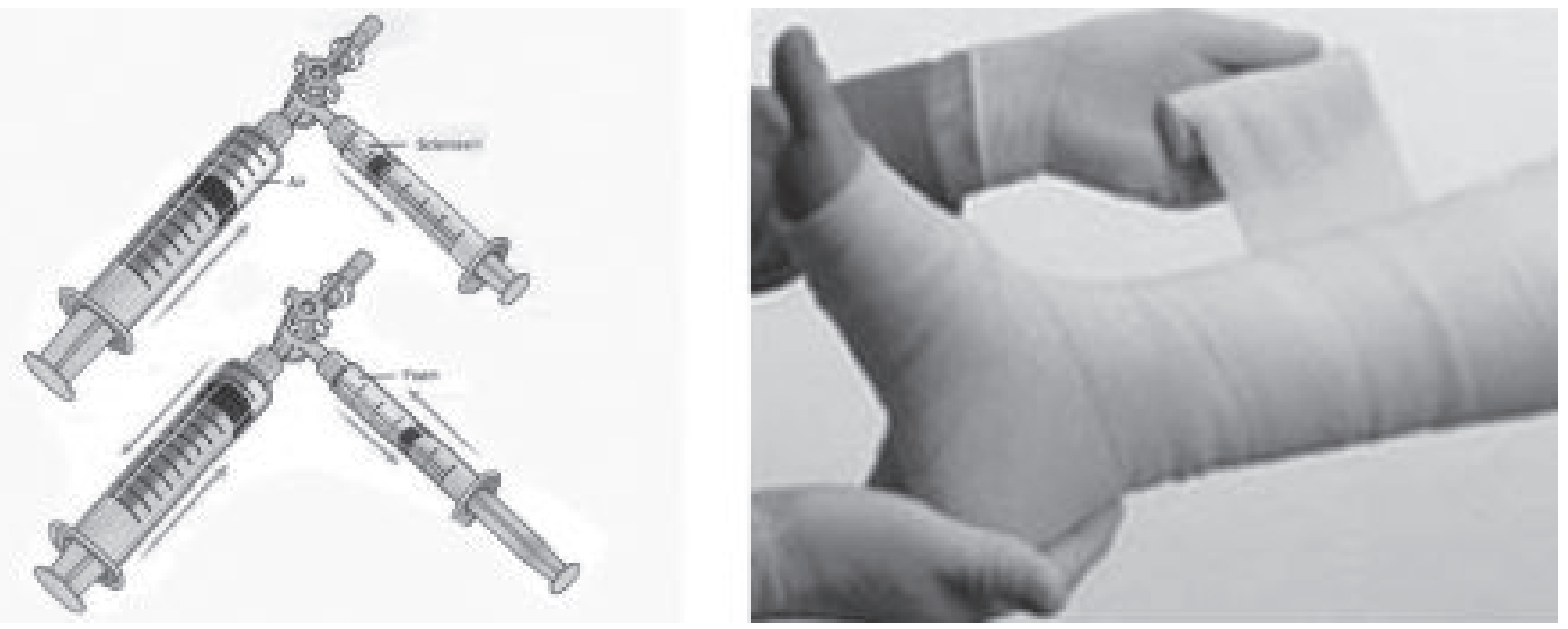

Fig.-3: A. Technique of mixing of foam sclerotherapy \& 3B. Application of crepe bandage

\section{Results:}

Vast majority of the patients had a smooth recovery. Significant pain was present in only $10 \%$ of patients for 1 week after laser therapy and $3 \%$ of patients noted bruising in thigh for 2 weeks. Among the 118 patients who reported for follow up at 12 months, $113(96 \%)$ showed effective persistent occlusion of GSV.

Table-I

Outcome of laser sclerotherapy

\begin{tabular}{lcc}
\hline Variable & $\mathrm{N}$ & $\%$ \\
\hline Total no. of case & 220 & \\
Total no. of limb & 297 & \\
Significant pain after 1 wk & & \\
Bruise at 2 wks & 7 & 3 \\
Wound infec within 1 wk & 6 & 2.7 \\
Persistent occlusion of GSV & 113 & 96 \\
\hline
\end{tabular}

\section{Discussion:}

Symptomatic lower extremity varicose vein is the most common vascular condition in adult population. Endovenous laser ablation (EVLA) is a promising minimally invasive treatment option for varicose vein. Modern Laser cath Lab with Laser Machine, Laser fibre, Duplex machine and highly competent manpower is neededso some what costly in our perspective. EVLA become established as a first line treatment option for varicose vein offered by vascular surgeon in comparison with traditional surgery.

\section{Bilateral Varicose Vein}

In this study, we have shown our experience in managing symptomatic varicose vein. All cases included were symptom of dilated and tortuous veins, active venous stasis ulcer and with clear symptoms of healed leg ulcer etc. Beside the clinical presentation, diagnosis and operative indication were confirmed by duplex study.

Over a 2 year period, patients who were referred by their Doctors with symptomatic varicose vein underwent duplex study of the lower limb veins. Patients with superficial truncal reflux $>0.5$ seconds and normal deep venous system were offered EVLA with $980 \mathrm{~nm}$ laser. Different wave length of laser fiber are available like 810, 940, 980, 1064, 1320, $1470 \mathrm{~nm}$. Clinical implication of different wave lengths is that more wave length laser fiber, more tissue penetration causing less bruising and less post operative analgesia is needed. Explanation is that more wave length is absorbed by intracelluar water and less wave length is absorved by haemoglobin. All procedures were performed under SAB with tumescent anesthesia along the course of the truncal vein. Concomitant foam sclerotherapy were also given in varicose vein at the same setting. During EVLA, treatment of incompetent perforator with Foam sclerotherapy is sufficient to destroy the incompetent perforatorts, so no need of conventionally treatment of subfascial ligation of incompetent perforators. Few contraindication of EVLA is only hugely dilated GSV and GSV is too close to the skin. 
A total 220 patients (297 limbs) were treated with EVLA from October 2013 to October 2015. Vast majority of the patients had a smooth recovery. Puncture site wound infection was $2.7 \%$ that was negligible. Significant pain was present in only $10 \%$ of patients for 1 week after laser therapy and $3 \%$ of patients noted bruising in thigh for 2 weeks. Among the 118 patients who reported for follow up at 12 months, 113 (96\%) showed effective persistent occlusion of GSV.

In this study, success was defined as complete relief of symptoms postoperatively like dull ache pain, heaviness of leg, healing of non-healing ulcer and improvement from leg discoloration that is lipodermatosclerosis, which has been achieved in all patients immediately or gradually during follow-up while respecting the difference between this outcome and other postoperative complications like numbness, hematoma, bruising. There is no deep vein thrombosis in our series but literature revealed that $1 \%$ chance of deep vein thrombosis In the evaluation of these results, certain limitations merit emphasis like duration of follow up is less, study sample is small and all patient not come for follow up. The study is a retrospective in nature and the data was collected from single center. However, this study presents the first reported experience of laser ablation of varicose vein in Bangladesh.

\section{Conclusions:}

High ligation, high ligation and stripping of GSV with multiple phlebectomy are morbid procedure and because of aggressive neovascularization at saphenofemoral junction bed, have high recurrence rate. Hematoma, wound infection, bruising, post operative pain and delayed return to normal activities are more prominent in high ligation and stripping than endovenous laser technique. As endovenous laser is a less invasive, cosmetic procedure, less post operative pain, require less hospital stay and ensure quick return to normal activities, so that its acceptance by the patient as well as by the vascular surgeon is increasing dramatically. It would therefore appear that endovenous laser ablation of GSV has established itself as a "new gold" standard for the treatment of varicose vein.

\section{Conflict of Interest - None.}

\section{References:}

1. Johnson CM, McLafferty RB. Endovenous laser ablation of varicose veins: review of current technologies and clinical outcome. Vascular 2007 sep-oct; 15(5): 250-254.

2. Niedzwiecki G, M.D. Endovenous Thermal Ablation of the Saphenous Vein. Seminars in Interventional Radiology 2005; 22(3):204-208.

3. Jones L, Braithwaite BD, Selwyn D, Cooke S, Earnshaw JJ. Neovascularization is the principal cause of varicose vein recurrence: results of a randomized trial of stripping the long saphenous vein. Eur J Vasc Endovasc Surg 1996; 12:442-445.

4. Dwerryhouse S, Davies B, Harradine K, Earnshaw JJ. Stripping the long saphenous vein reduces the rate of reoperation for recurrence varicose veins. J Vasc Surg 1999; 29:589-592.

5. Fischer R, Linde N, Dff C, Jeanneret C, Chandler JG, Seeber P. Late recurrent saphenofemoral junction reflux after ligation and stripping of the great saphenous vein. $\mathrm{J}$ Vasc Surg 2001; 34:236-240.

6. Mackenzie RK, Paisley A, Allan PL, Lee AJ, Ruckley CV, Bradbury AW. The effect of long saphenous vein stripping on quality of life. J Vasc Surg 2002; 35:1197-1203.

7. Mackey DC, Summerton DJ, Walker AJ. The early morbidities of varicose vein surgery. J R Nav Med Serv 1995; 81:42-46.

8. Rutgers PH, Kitslaar PJ. Randomized trial of stripping versus high ligation combined with sclerotherapy in the treatment of the incompetent greater saphenous vein. Am J Surg 1994; 168:311-315.

9. Davies AH, Shepherd AC, Gohel MS. Endovenous laser ablation: Experiences from the United Kingdom 2008/2009. Argentina, April 21-25 ${ }^{\text {th }}, 2010$. 